\title{
Doenças Crônicas Não Transmissíveis e fatores de risco e proteção em adultos com ou sem plano de saúde
}

\author{
Noncommunicable diseases, risk factors, and protective factors in \\ adults with and without health Insurance
}

\author{
Deborah Carvalho Malta (https://orcid.org/0000-0002-8214-5734) ${ }^{1}$ \\ Regina Tomie Ivata Bernal (https://orcid.org/0000-0002-7917-3857) ${ }^{1}$ \\ Eduardo Vieira Neto (https://orcid.org/0000-0002-0247-837X) ${ }^{2}$ \\ Kátia Audi Curci (https://orcid.org/0000-0001-9476-0542) ${ }^{2}$ \\ Maria Tereza de Marsillac Pasinato (https://orcid.org/0000-0002-8209-894X) ${ }^{2}$ \\ Raquel Medeiros Lisbôa (https://orcid.org/0000-0002-0159-0044) ${ }^{2}$ \\ Renata Fernandes Cachapuz (https://orcid.org/0000-0001-9970-4830) ${ }^{2}$ \\ Karla Santa Cruz Coelho (https://orcid.org/0000-0003-4943-4814) ${ }^{3}$ \\ Fausto Pereira dos Santos (https://orcid.org/0000-0001-7100-6918) ${ }^{4}$ \\ Maria Imaculada Fátima de Freitas (https://orcid.org/0000-0002-0273-9066) ${ }^{1}$
}

${ }^{1}$ Escola de Enfermagem, Universidade Federal de Minas Gerais. Av. Professor Alfredo Balena 190, Santa Efigênia. 30130-100 Belo Horizonte MG Brasil. dcmalta@uol.com.br

${ }^{2}$ Diretoria de Normas e Habilitação dos Produtos, Agência Nacional de Saúde Suplementar. Brasília DF Brasil.

${ }^{3}$ Universidade Federal do Rio de Janeiro. Macaé RJ Brasil.

${ }^{4}$ Instituto René Rachou, Fiocruz. Belo Horizonte MG Brasil.

\begin{abstract}
This study describes the coverage of health insurance and compares the occurrence of risk factors $(R F)$ and protective factors of noncommunicable diseases in the population with and without health insurancesin Brazilianstate capitals. Data from the telephone survey Vigitel was analyzed. The Poisson regression model was used to estimate the prevalence ratio (PR), comparing RF among those who did or did not have a health insurance. Plan coverage was $49.1 \%$, and the highest prevalences were in Goiania, Vitoria, Florianópolis, and Belo Horizonte. Adults over 55 years of age and with higher education were more likely to have an insurance. The population with health insurance hada higher prevalence of protective factors, such as fruit and vegetable consumption ( $P R=1.3$ 95\% CI 1.2-1.3), physical activity in their free time $(P R=1.2(95 \%$ CI: 1.2-1.3), mammographies ( $R P=1.2$ IC95\% 1.1-1.3) and pap smears $(P R=1.1$ IC95\% 1.21.3), and lower prevalence of RFs such as smoking $(R P=0.7,95 \%$ CI 0.6-0.8), poor health $(R P=$ 0.8 CI95\% 0.6-0.9), obesity ( $R P=0.8$ IC95\% 0.7-0.9), consumption of meat with fat $(R P=0.9$ IC95\% 0.8-0.9) and whole milk (RP = 0.9 IC95\% 0,8-0.9). Regardless of educational level, the population that has health insurancesgenerally has better indicators, such as healthier habits and greater coverage of preventive exams.
\end{abstract}

Key words Health insurance, Chronic diseases, Hypertension, Healthy eating, Physical activity
Resumo O estudo descreve as coberturas de planos de saúde e compara a ocorrência de fatores de risco (FR) e proteção de Doenças Crônicas Não Transmissíveis, na população com e sem planos de saúde nas capitais brasileiras. Foram analisados dados do inquérito telefônico Vigitel. Foi utilizado o modelo de regressão de Poisson para estimar a razão de prevalência ( $R P)$, comparando FR entre quem tem ou não plano de saúde. A cobertura de planos foi de 49,1\%, mais elevada em Goiania, Vitória, Florianópolis e Belo Horizonte, entre adultos acima de 55 anos e com maior escolaridade. A população com planos de saúde apresenta prevalências mais elevadas de fatores de proteção como consumo de frutas e hortaliças ( $R P=1,3$ IC95\% 1,2-1,3), prática de atividade física no tempo livre $(R P=1,2$ IC95\% 1,2-1,3), mamografia $(R P=1,2$ IC95\% 1,1-1,3) e Papanicolau ( $R P=1,1$ IC95\% 1,2-1,3), e menor prevalência de FR como tabagis$m o$ ( $R P=0,7$ IC95\% 0,6-0,8), avaliação de saúde ruim $(R P=0,8$ IC95\% 0,6-0,9), obesidade ( $R P$ $=0,8$ IC95\% 0,7-0,9), carne com gordura $(R P=$ 0,9 IC95\% 0,8-0,9) e leite com gordura $(R P=0,9$ IC95\% 0,8-0,9). Independentemente da escolaridade, a população que tem planos de saúde apresenta geralmente, melhores indicadores, como hábitos mais saudáveis e maior cobertura de exames preventivos.

Palavras-chave Planos de saúde, Doenças crônicas, Hipertensão, Alimentação saúdavel, Atividade física 


\section{Introdução}

As doenças cardiovasculares, o câncer, a diabetes e as doenças respiratórias crônicas são as principais doenças crônicas não transmissíveis (DCNT), responsáveis por cerca de $70 \%$ de todas as mortes no mundo. Essas doenças resultam em mortes prematuras, perda de qualidade de vida, além de resultar em impactos econômicos negativos para as famílias, os indivíduos e a sociedade. O aumento da carga de DCNT reflete os efeitos negativos da urbanização rápida e da globalização, que induzem a maioria dos países a estilos de vida sedentários, alimentação com alto teor calórico e com alimentos ultraprocessados, além do uso do tabaco e do álcool ${ }^{1,2}$. Em geral, as DCNT afetam com maior frequência populações de baixa renda, por estarem mais expostas aos fatores de risco e terem menor acesso aos serviços de saúde ${ }^{1-4}$.

Estudos apontam desigualdades na distribuição da morbimortalidade das DCNT e seus fatores de risco, segundo fatores socioeconômicos, como educação, ocupação, renda, gênero e etnia ${ }^{1,3}$, escolaridade, posse de planos de saúde ${ }^{4-6}$. A literatura descreve também uma correlação positiva entre acesso aos serviços de saúde, escolaridade e renda da população ${ }^{7-9}$. Dados da Pesquisa Nacional de Saúde (PNS) apontaram que quanto maior a escolaridade do chefe da família, maior sua chance de ter um plano de saúde. Ocorreu incremento nessas proporções, considerando-se que, da população com nível superior completo, $61,7 \%$ possuem planos de saúde, e da população com menor escolaridade ( 0 a 8 anos de estudo), $14,1 \%$ relataram também o possuírem, independentemente do tipo de plano ${ }^{10,11}$. A mesma pesquisa apontou que a população com maior escolaridade e com planos de saúde teve mais acesso a serviços de saúde ${ }^{6}$, sejam privados ou públicos, a exames preventivos de câncer, como mamografia $^{12}$, além de menor frequência de DCNT e menor incapacidade decorrentes destas doenças ${ }^{6}$.

Estudos americanos com dados do Behavior Risk Factor Surveillance System (BRFSS) apontam que populações com planos de saúde tendem a ter mais acesso a exames preventivos, maiores prevalências de fatores de proteção e menor prevalência de fatores de risco ${ }^{13,14}$. Estes estudos relacionam tais resultados com maior escolaridade e melhores condições sociais ${ }^{13,14}$. No Brasil, ainda são poucos estudos comparativos entre a população com e sem planos de saúde ${ }^{15}$. Inquéritos realizados em Belo Horizonte apontaram diferenças entre essas populações, sempre associando maio- res prevalências de fatores de risco a populações não cobertas por planos de saúde ${ }^{8}$.

A partir de 2006, o Sistema Nacional de Vigilância de Doenças Crônicas por Inquérito Telefônico (Vigitel) passou a monitorar as DCNT e seus fatores de risco e, em 2008, foi incluída a variável plano de saúde. São poucos os estudos nacionais que comparam as diferentes populações com e sem plano. Considerando a constante mudança nas coberturas de planos de saúde no Brasil ${ }^{10}$, e o aumento na população coberta por planos de Saúde no país, segundo dados da Pesquisa Nacional de Saúde em $2013^{11}$, torna-se importante este tipo de estudo para apontar desigualdades em saúde e apoiar a superação das mesmas.

Neste artigo buscou-se analisar as coberturas de planos de saúde no Brasil e comparar a ocorrência de fatores de risco e proteção de DCNT, morbidade referida e acesso a exames preventivos na população com e sem planos de saúde, no conjunto das capitais brasileiras.

\section{Métodos}

No presente estudo, de corte transversal, analisou-se dados do Vigitel para a população adulta ( $\geq 18$ anos) residente nas capitais dos 26 estados brasileiros e no Distrito Federal no ano de 2015. O Vigitel utilizou amostras probabilísticas de linhas de telefone fixo das cidades, disponibilizadas pelas principais operadoras de telefonia fixa no país. Foram sorteadas, de forma aleatória, 5.000 linhas telefônicas de cada capital, que foram divididas em réplicas (ou subamostras) de 200 linhas cada, para identificação de linhas residenciais ativas, consideradas elegíveis para a pesquisa. Para cada linha eleita foi realizado sorteio de um dos adultos moradores do domicílio, para ser entrevistado ${ }^{16,17}$.

Foi utilizado o método raking ${ }^{18,19}$ para o cálculo dos pesos de pós-estratificação, com base em fonte externa de dados da população brasileira. $\mathrm{Na}$ construção dos pesos de pós-estratificação foram usadas estimativas do Instituto Brasileiro de Geografia e Estatistica (IBGE) de idade, sexo e escolaridade da população projetada para o ano corrente da pesquisa ${ }^{17}$. O método raking utiliza a distribuição de frequências absolutas de faixa etária $(18$ a $24 ; 25$ a $34 ; 35$ a $44 ; 45$ a $54 ; 65$ anos ou mais), sexo (masculino, feminino) e nível de instrução ( 0 a 7; 8 a 10; 11 a 13; 14 anos ou mais) da população, ponderada pelos pesos amostrais. Os pesos foram calculados no programa SAS, utilizando a macro sasRakinge.sas disponibilizada por Izrael et al. ${ }^{19}$. 
O questionário do Vigitel engloba 94 questões, divididas em módulos: Características demográficas e socioeconômicas dos indivíduos; padrão de alimentação e atividade física; peso e altura referidos; consumo de cigarro e de bebidas alcoólicas; avaliação própria do seu estado de saúde, morbidade referida e exames preventivos.

No estudo atual, foram analisadas a cobertura de quem refere ter plano de saúde nas capitais brasileiras e a Razão de Prevalência da posse de planos por sexo e faixa etária na população com plano. As prevalências, com os respectivos IC95\%, foram comparadas nas variáveis relativas ao tabagismo (fumantes, ex-fumantes, fumantes passivos no domicílio, fumantes passivos no trabalho); e ao peso corporal (excesso de peso - Índice de Massa Corporal $\geq 25 \mathrm{~kg} / \mathrm{m}^{2}$; obesidade Índice de Massa Corporal $\geq 30 \mathrm{~kg} / \mathrm{m}^{2}$ ). Os valores ausentes de excesso de peso e obesidade sofreram imputação, conforme metodologia empregada pelo Vigitel e descrita previamente ${ }^{17}$. As demais prevalências calculadas foram relativas ao consumo de carnes com excesso de gordura (carne vermelha com gordura visível ou frango com pele); consumo de leites com teor integral de gorduras; consumo regular de refrigerantes ou suco artificial (cinco ou mais dias por semana); consumo de doces (cinco ou mais dias por semana); inatividade física (indivíduos que não praticaram qualquer atividade física no tempo livre nos últimos três meses, que não realizam esforços físicos intensos no trabalho, que não se deslocam para o trabalho ou escola a pé ou de bicicleta e que não são responsáveis pela limpeza pesada de suas casas); $\mathrm{AF}<150$ minutos nos dominios do tempo livre, trabalho e deslocamento; hábito de assistir TV; consumo abusivo de bebidas alcoólicas (quatro ou mais doses para mulher e cinco ou mais doses para homem) em uma mesma ocasião nos últimos 30 dias, considerando como dose de bebida alcoólica uma dose de bebida destilada, uma lata de cerveja ou uma taça de vinho); direção de veículo motorizado após consumo de qualquer quantidade de bebida alcoólica; autoavaliação do estado de saúde ruim; e morbidades referidas (diagnóstico médico prévio de hipertensão arterial e diabetes).

Os fatores de proteção apresentados foram: consumo recomendado (cinco ou mais porções diárias, em cinco ou mais dias da semana) de frutas e hortaliças; consumo regular de feijão (em cinco ou mais dias da semana); prática recomendada de atividade física no tempo livre (pelo menos 150 minutos semanais de atividade física de intensidade leve ou moderada, ou de, pelo menos, 75 minutos semanais de atividade física de inten- sidade vigorosa, independentemente do número de dias em que pratica atividade física por semana); realização de exames de detecção precoce de câncer em mulheres (mamografia para mulheres de 50 a 69 anos, nos últimos dois anos, e exame de Papanicolau para mulheres de 25 a 59 anos, nos últimos três anos).

Tais indicadores foram calculados tendo como denominador o total de adultos entrevistados, à exceção daqueles referentes à idade e sexo específicos. As prevalências foram calculadas ponderando-se para ajustar a distribuição sociodemográfica da amostra Vigitel à distribuição da população adulta da cidade, segundo aspectos Metodológicos já descritos ${ }^{17}$, sendo o cálculo das RP realizado com ajuste por idade, sexo e escolaridade, entre usuários ou não de planos de saúde, com base no modelo de Poisson ${ }^{20}$.

Foram ainda comparados os fatores de risco entre a população com ou sem plano, segundo a prevalência, Razão de Prevalência ajustada por idade e sexo, e estratificada segundo a escolaridade de 0 a 8 anos, 9 a 11 anos e 12 anos e mais de estudo.

$\mathrm{O}$ inquérito Vigitel foi aprovado pela Comissão Nacional de Ética em Pesquisa para Seres Humanos, do Ministério da Saúde. A assinatura do Termo de Consentimento Livre e Esclarecido, neste inquérito foi substituída pelo consentimento verbal do entrevistado no momento da ligação telefônica.

\section{Resultados}

No conjunto das capitais, a cobertura de planos foi de $49,1 \%$, variando entre $30,4 \%$ Rio Branco a 64,6\% em Goiânia (Tabela 1).

Tendo como referência a população de 18 a 24 anos, a faixa etária de 25 a 34 anos teve menor $\mathrm{RP}$ de posse de plano de saúde ( $\mathrm{RP}=0,9$ IC95\% 0,8-0,99), as maiores RP foram nas faixas de 55 a 64 anos ( RP $=1,1$ IC95\% 1,05-1,2) e de 65 anos e mais $(\mathrm{RP}=1,4$ IC95\% 1,3-1,5). Quanto maior a escolaridade houve maior chance de ter planos - 9 a 11 anos (RP = 1,7 IC95\% 1,6-1,8) e 12 a 20 anos ( $\mathrm{RP}=2,8$ IC95\% 2,6-2,9) (Tabela 2).

A distribuição dos fatores de risco e proteção de Doenças Crônicas Não Transmissíveis com e sem de planos de saúde, no conjunto das $26 \mathrm{ca}-$ pitais, e as RP estimadas estão apresentadas na Tabela 3. Em geral, prevalências mais elevadas de fatores de proteção e menores frequências de fatores de risco foram encontradas na população com planos de saúde. 
Tabela 1. Tamanho da amostra e cobertura de plano de saúde por capital. Brasil, Vigitel, 2015.

\begin{tabular}{|c|c|c|c|c|c|c|}
\hline \multirow{2}{*}{ Cidade } & \multicolumn{2}{|c|}{ Ter planos } & \multirow{2}{*}{$\begin{array}{c}\text { Amostra } \\
\text { Total }\end{array}$} & \multicolumn{3}{|c|}{ Posse planos } \\
\hline & Não & Sim & & $\%^{(*)}$ & & \\
\hline Aracaju & 788 & 1.211 & 1.999 & 52,11 & 49,25 & 54,98 \\
\hline Belém & 1.005 & 993 & 1.998 & 43,27 & 40,60 & 45,95 \\
\hline Belo Horizonte & 701 & 1.303 & 2.004 & 60,73 & 58,06 & 63,39 \\
\hline Boa Vista & 1.084 & 932 & 2.016 & 31,54 & 28,49 & 34,59 \\
\hline Campo Grande & 1.097 & 904 & 2.001 & 43,29 & 40,55 & 46,03 \\
\hline Cuiába & 561 & 1.440 & 2.001 & 57,67 & 53,74 & 61,59 \\
\hline Curitiba & 918 & 1.079 & 1.997 & 53,77 & 51,16 & 56,39 \\
\hline Florianópolis & 550 & 1.445 & 1.995 & 61,76 & 58,51 & 65,01 \\
\hline Fortaleza & 1.037 & 953 & 1.990 & 43,03 & 40,31 & 45,76 \\
\hline Goiânia & 378 & 1.618 & 1.996 & 64,59 & 60,18 & 69,01 \\
\hline João Pessoa & 1.017 & 974 & 1.991 & 34,79 & 32,11 & 37,48 \\
\hline Macapá & 923 & 1.070 & 1.993 & 41,10 & 37,71 & 44,50 \\
\hline Maceió & 1.136 & 860 & 1.996 & 38,93 & 36,12 & 41,73 \\
\hline Manaus & 1.061 & 939 & 2.000 & 38,41 & 35,01 & 41,81 \\
\hline Natal & 962 & 1.052 & 2.014 & 42,80 & 39,90 & 45,71 \\
\hline Palmas & 722 & 1.272 & 1.994 & 49,93 & 46,62 & 53,25 \\
\hline Porto Alegre & 953 & 1.051 & 2.004 & 50,81 & 47,77 & 53,86 \\
\hline Porto Velho & 827 & 1.172 & 1.999 & 41,97 & 38,61 & 45,34 \\
\hline Recife & 1.124 & 879 & 2.003 & 38,83 & 36,23 & 41,42 \\
\hline Rio Branco & 1.110 & 891 & 2.001 & 30,39 & 27,44 & 33,35 \\
\hline Rio de Janeiro & 631 & 1.373 & 2.004 & 53,91 & 50,51 & 57,30 \\
\hline Salvador & 975 & 1.019 & 1.994 & 46,66 & 43,72 & 49,59 \\
\hline São Luís & 1.191 & 805 & 1.996 & 35,19 & 32,36 & 38,03 \\
\hline São Paulo & 923 & 1.075 & 1.998 & 48,01 & 45,37 & 50,65 \\
\hline Teresina & 828 & 1.164 & 1.992 & 47,95 & 44,94 & 50,96 \\
\hline Vitória & 554 & 1.438 & 1.992 & 64,29 & 61,16 & 67,42 \\
\hline Distrito Federal & 366 & 1.637 & 2.003 & 59,85 & 55,50 & 64,20 \\
\hline Total & 23.422 & 30.549 & 53.971 & 49,12 & 48,18 & 50,06 \\
\hline
\end{tabular}

Nota: $\left(^{*}\right.$ Percentual ponderado para ajustar a distribuição sociodemográfica da amostra Vigitel à distribuição da população adulta da cidade projetada para cada ano da pesquisa.

Tabela 2. Razão de Prevalência da posse de planos de saúde nas capitais brasileiras, segundo idade e escolaridade. Brasil, Vigitel, 2015

\begin{tabular}{cccc}
\hline Variável & RP & \multicolumn{2}{c}{ IC(95\%) } \\
\hline Faixa etária & & & \\
18 a 24 & 1,00 & & \\
25 a 34 & 0,93 & 0,88 & 0,99 \\
35 a 44 & 0,97 & 0,91 & 1,03 \\
45 a 54 & 1,07 & 1,00 & 1,14 \\
55 a 64 & 1,12 & 1,05 & 1,20 \\
65 e mais & 1,42 & 1,34 & 1,52 \\
Escolaridade & & & \\
0 a 8 & 1,00 & & \\
9 a 11 & 1,71 & 1,60 & 1,84 \\
12 a 20 & 2,76 & 2,59 & 2,95
\end{tabular}

Na população com plano foram encontradas menores prevalências de tabagismo - 7,7\% (IC $95 \%$ 6,9-8,5), de ex-fumantes, de consumo pesado de fumo, ou 20 ou mais cigarros ao dia, e de fumantes passivos no trabalho e domicílio. $\mathrm{O}$ excesso de peso ocorreu em mais da metade da população com planos - 51,6\% (IC95\% 50,3-52,8), embora tenha sido mais elevado na população sem planos - 56,2\% (IC95\% 54,8-57,6), bem como a obesidade - 16,5\% (IC95\% 15,6-17,4), e sem planos - 21,3\% (IC95\% 20,1-22,5).

Em relação ao consumo alimentar, houve melhores frequências de indicadores saudáveis na população com planos, como o recomendado de frutas e hortaliças - 30,9\% (IC95\% 29,8-32,1), e menores frequências de indicadores não sau- 
Tabela 3. Fatores de risco e proteção de Doenças Crônicas Não Transmissíveis com e sem de planos de saúde nas capitais Brasileiras Brasil, Vigitel, 2015.

\begin{tabular}{|c|c|c|c|c|c|c|c|c|c|}
\hline \multirow{3}{*}{ Variável } & \multicolumn{6}{|c|}{ Posse de Plano de Saúde } & \multirow{3}{*}{$\mathrm{RPaj}^{*}$} & \multirow{2}{*}{\multicolumn{2}{|c|}{ IC 95\% }} \\
\hline & \multicolumn{3}{|c|}{ Sim } & \multicolumn{3}{|c|}{ Não } & & & \\
\hline & \multirow{2}{*}{$\begin{array}{r}\text { Prev } \\
7,7\end{array}$} & \multicolumn{2}{|c|}{ IC $95 \%$} & \multirow{2}{*}{$\begin{array}{c}\text { Prev } \\
13,0\end{array}$} & \multicolumn{2}{|c|}{ IC $95 \%$} & & & \\
\hline Fumante & & 6,9 & 8,5 & & 11,9 & 14,0 & 0,7 & 0,6 & 0,8 \\
\hline Ex-fumante & 18,9 & 18,0 & 19,8 & 22,6 & 21,4 & 23,7 & 0,6 & 0,5 & 0,9 \\
\hline 20 ou mais cigarros & 2,1 & 1,6 & 2,5 & 4,1 & 3,4 & 4,8 & 0,9 & 0,8 & 0,98 \\
\hline Fumantes passivos no domicílio & 7,9 & 7,2 & 8,7 & 10,2 & 9,3 & 11,2 & 0,8 & 0,7 & 0,9 \\
\hline Fumantes passivos trabalho & 6,7 & 5,9 & 7,4 & 9,3 & 8,4 & 10,2 & 0,8 & 0,7 & 0,9 \\
\hline Excesso de peso & 51,6 & 50,3 & 52,8 & 56,2 & 54,8 & 57,5 & 1,0 & 0,9 & 1,0 \\
\hline Obesidade & 16,5 & 15,6 & 17,4 & 21,3 & 20,1 & 22,5 & 0,8 & 0,7 & 0,9 \\
\hline Regular FH & 44,7 & 43,4 & 45,9 & 30,9 & 29,6 & 32,1 & 1,3 & 1,2 & 1,4 \\
\hline Recomendado de FH & 30,9 & 29,8 & 32,1 & 19,8 & 18,7 & 20,9 & 1,3 & 1,2 & 1,4 \\
\hline Carnes com gordura & 28,3 & 27,1 & 29,5 & 33,9 & 32,5 & 35,3 & 0,9 & 0,8 & 0,9 \\
\hline Leite com gordura & 46,7 & 45,4 & 47,9 & 56,1 & 54,7 & 57,5 & 0,9 & 0,8 & 0,9 \\
\hline Refrigerantes ( $5 \mathrm{x}$ ou mais/Sem) & 17,7 & 16,7 & 18,8 & 20,2 & 18,9 & 21,4 & 0,9 & 0,8 & 1,0 \\
\hline Doces $(5 \mathrm{x}$ ou mais/Sem $)$ & 22,8 & 21,7 & 23,9 & 17,4 & 16,3 & 18,5 & 1,2 & 1,1 & 1,3 \\
\hline Feijão ( $5 x$ ou mais/Sem) & 60,6 & 59,4 & 61,8 & 68,8 & 67,5 & 70,0 & 0,9 & 0,9 & 1,0 \\
\hline AF no lazer & 43,9 & 42,6 & 45,1 & 31,7 & 30,4 & 33,0 & 1,2 & 1,2 & 1,3 \\
\hline Inatividade Física & 16,0 & 15,1 & 16,9 & 15,9 & 14,9 & 17,0 & 1,1 & 1,0 & 1,2 \\
\hline $\mathrm{AF} \geq=150 \mathrm{~min} / \mathrm{sem} 3$ domínios & 55,3 & 54,0 & 56,5 & 49,9 & 48,5 & 51,3 & 1,1 & 1,0 & 1,1 \\
\hline $\mathrm{AF}<150 \mathrm{~min} / \mathrm{sem} 3$ domínios & 44,7 & 43,5 & 46,0 & 50,1 & 48,7 & 51,5 & 0,9 & 0,9 & 1,0 \\
\hline Assistir TV - 3hrs/dia & 20,9 & 19,9 & 21,9 & 24,2 & 22,9 & 25,4 & 0,9 & 0,8 & 1,0 \\
\hline Consumo abusivo de álcool & 17,1 & 16,1 & 18,0 & 17,3 & 16,2 & 18,5 & 0,9 & 0,8 & 1,0 \\
\hline Direção após consumo de álcool & 6,6 & 6,0 & 7,2 & 4,5 & 3,9 & 5,1 & 1,1 & 0,9 & 1,3 \\
\hline Avaliação ruim de saúde & 3,5 & 3,0 & 4,1 & 6,0 & 5,3 & 6,7 & 0,8 & 0,6 & 0,9 \\
\hline Mamografia últimos dois anos & 87,1 & 85,1 & 89,0 & 70,3 & 67,8 & 72,9 & 1,2 & 1,1 & 1,2 \\
\hline Papanicolau nos últimos três anos & 86,0 & 84,6 & 87,4 & 76,5 & 74,8 & 78,2 & 1,1 & 1,1 & 1,2 \\
\hline Hipertensão & 22,5 & 21,5 & 23,5 & 27,2 & 26,0 & 28,4 & 0,9 & 0,8 & 0,96 \\
\hline Diabetes & 6,7 & 6,1 & 7,3 & 8,1 & 7,3 & 8,8 & 0,9 & 0,8 & 1,1 \\
\hline
\end{tabular}

${ }^{*}$ Razão de prevalência ajustada por idade, sexo e escolaridade.

dáveis, como carnes gordurosas - 28,3 (IC95\% 27,1-29,5), leite integral, com gordura - 46,7\% (IC95\% 45,4-47,9) e refrigerantes - 17,7 (IC95\% 16,7-18,8). As exceções foram o maior consumo de doces e o menor consumo de feijão pela população com planos de saúde.

A prática de atividade física no lazer foi mais elevada na população com planos - 43,9\% (IC95\% 42,6-45,1); a inatividade física não mostrou diferenças entre as duas populações; assistir e número de horas de TV foi menor entre quem tem planos.

O consumo abusivo de álcool foi de $17,1 \%$ (IC95\% 16,1-18,0) e dirigir após consumo de bebida alcoólica foi mais elevado na população com planos. A autoavaliação do estado de saúde ruim foi mais baixa na população com planos 3,53 (IC95\% 2,98-4,1) comparada com população sem planos 6,0\% (IC95\% 5,3-6,7) e as prevalências de morbidades autoreferidas (hipertensão e diabetes) também foram menores entre os que têm plano de saúde.

Os exames preventivos de câncer em mulheres, cobertura de mamografia e Papanicolau, foram mais elevados na população com planos: de 87,0 (IC95\% 85,1-89) e 86,0 (IC95\% 84-87,4), sem planos (Tabela 3 ).

Após ajustamento por idade, sexo e escolaridade, ter plano de saúde foi associado à menor prevalência de tabagismo (RP 0,70, IC95\% 0,6$0,8)$, de ser ex-fumante $(\mathrm{RP}=0,6$ IC95\% 0,5 $0,9)$, ter consumo de 20 ou mais cigarros ao dia 
$(\mathrm{RP}=0,9 \mathrm{IC} 95 \% 0,8-0,98)$, fumantes passivos no domicílio e no trabalho ( $\mathrm{RP}=0,8$ IC95\% 0,7-0,9 e 0,8 IC95\% 0,7-0,9 respectivamente).

Após os mesmos ajustamentos, o fato de ter plano de saúde também foi associado à menor obesidade ( $\mathrm{RP}=0,8$ IC95\% 0,7-0,9), ao maior consumo de frutas e hortaliças, tanto regular (RP $=1,3$ IC95\% 1,2-1,4) como recomendado $(\mathrm{RP}=$ 1,3 IC95\% 1,2-1,4), ao menor consumo de carnes com gorduras $(\mathrm{RP}=0,9 \mathrm{IC} 95 \% 0,8-0,9) \mathrm{e}$ de leite com teor integral de gordura ( $\mathrm{RP}=0,9$ IC95\% 0,8-0,9). Não houve diferenças na associação com consumo de refrigerantes em cinco ou mais dias da semana; mas ocorreu maior consumo de doces entre usuarios de planos (RP 1,2 IC95\% 1,1-1,3) e menor consumo de feijão em cinco ou mais dias da semana ( $\mathrm{RP}=0,9$ IC95\% $0,8-1,0)$. A avaliação de estado de saúde considerada como ruim foi menor em usuários de plano $(\mathrm{RP}=0,8 \mathrm{IC} 95 \% 0,6-0,9)$ e o RP foi menor para hipertensão arterial, embora dentro do IC95\%. A atividade física no tempo livre foi maior no grupo dos que possuem plano de saúde $(\mathrm{RP}=1,2$ IC95\% 1,2-1,3), enquanto os demais indicadores de AF encontram-se no limite do IC95\%. Não houve diferenças nas variáveis relativas a consumo abusivo de álcool e condução de veículo motorizado após ingerir álcool entre as duas populações com e sem plano de saúde. Os exames preventivos, como mamografia em mulheres entre 50 e 69 anos de idade nos dois últimos anos, e Papanicolau em mulheres entre 25 e 64 anos de idade, nos três últimos anos, foram maiores entre mulheres com planos de saúde ( $\mathrm{RP}=1,2$ IC $95 \%$ $1,1-1,2)$ e (RP $=1,1$ IC 95\% 1,1-1,2), respectivamente (Tabela 3).

Na Tabela 4 encontra-se a RP dos dos fatores de risco e proteção de Doenças Crônicas Não Transmissíveis na população com e sem de plano de saúde, segundo três estratos de escolaridade ( 0 a 8 anos, 9 a 11 anos, 12 a 20 anos de escolaridade), ajustada por sexo e idade. Em geral, os indicadores foram melhores entre as pessoas que possuem plano de saúde. Em todos os estratos de escolaridade se verificou maior consumo regular e recomendado de frutas e hortaliças; menor consumo de leite com gorduras; maior RP para a prática de atividade física; maior cobertura de mamografia. No estrato de menor escolaridade (0 a 8 anos), entre quem tem planos, foi encontrado menor RP de consumo de leite com gordura e de obesidade. No estrato de 9 a 11 anos foi encontrada menor RP de fumante passivo no trabalho, menor RP de assistir TV 3 horas e mais, menor avaliação ruim de saúde, e menor RP de
Hipertensão. Nos estratos de 9 anos a 11 anos e 12 e mais de estudo, entre quem tem planos, foram encontradas menores RP de fumantes. No estrato de 12 anos e mais de escolaridade foi encontrado menor RP de consumo de carnes com gordura, consumo de feijão, maior RP de direção após consumo de álcool.

\section{Discussão}

O estudo mostra que cerca de metade da população nas capitais tem plano de saúde, com coberturas mais elevadas nas capitais do Sudeste, Sul e Centro Oeste. Dentre os Estados, Goiânia e Vitória tiveram coberturas acima de $64 \%$. Ter planos de saúde foi mais elevado em populações com escolaridade mais elevada e com 55 anos de idade e mais. Em geral, a população com planos de saúde apresenta prevalências mais elevadas de fatores de proteção como alimentação saudável (maior consumo de frutas e hortaliças), prática de atividade física no tempo livre, maior cobertura de exames preventivos, como mamografia e Papanicolau, além de menor prevalência de fatores de risco como tabagismo, inatividade física, avaliação de saúde ruim, hipertensão arterial, obesidade, consumo de bebida alcoólica de forma abusiva, consumo de carne com excesso de gordura, leite com gorduras e refrigerantes, embora tenha menor consumo de feijão e maior consumo de doces. Quando estratificadas por escolaridade, essas características, em geral, tendem a se manter, e a população que tem planos de saúde, independentemente da escolaridade, geralmente apresenta melhores indicadores.

As menores coberturas de planos de saúde nas capitais do Nordeste e Norte do país coincidem com as estimativas da ANS e da PNS ${ }^{10,11,21}$. Em geral, coberturas mais elevadas são frequentes em áreas urbanas, nas capitais, nos municípios mais populosos e em regiões com maior concentração de riquezas e atividade econômica ${ }^{7,10,11,21}$. As coberturas aqui identificadas são mais elevadas que os dados da PNS, que identificaram $40 \%$ de cobertura nas capitais e $27 \%$ na população geral ${ }^{7}$. A PNS também apontou crescimento da população com planos de saúde em relação a PNAD 2008 ${ }^{7,10}$. Os dados do Vigitel 2015 foram consistentes com o crescimento dos planos de saúde, que apresentava, em 2011, 47,4\% de cobertura ${ }^{15}$.

Outros estudos também apontam associação entre escolaridade e posse de um plano de saúde $8,11,13,22$, conforme aqui identificado. A PNS também mostra que a posse de planos está for- 
Tabela 4: Distribuição dos fatores de risco e proteção de Doenças Crônicas Não Transmissíveis na população com e sem de planos de saúde, segundo estratos de escolaridade no conjunto das 26 capitais e Distrito Federal. Brasil, Vigitel, 2015

\begin{tabular}{|c|c|c|c|c|c|c|c|c|c|c|c|c|c|c|c|}
\hline \multirow{4}{*}{ Variável } & \multicolumn{15}{|c|}{ Escolaridade } \\
\hline & \multirow{2}{*}{\multicolumn{5}{|c|}{$\begin{array}{c}0 \text { a } 8 \\
\text { Plano de saúde }\end{array}$}} & \multirow{2}{*}{\multicolumn{5}{|c|}{$\begin{array}{c}9 \text { a } 11 \\
\text { Plano de saúde }\end{array}$}} & \multirow{2}{*}{\multicolumn{5}{|c|}{$\frac{12+}{\text { Plano de saúde }}$}} \\
\hline & & & & & & & & & & & & & & & \\
\hline & \multirow[t]{2}{*}{ Sim } & \multirow[t]{2}{*}{ Não } & \multirow[t]{2}{*}{ Rpaj* $^{*}$} & \multicolumn{2}{|c|}{ IC95\% } & \multirow[t]{2}{*}{ Sim } & \multirow[t]{2}{*}{ Não } & \multirow[t]{2}{*}{ RPaj $^{*}$} & \multicolumn{2}{|c|}{ IC95\% } & \multirow[t]{2}{*}{ Sim } & \multirow[t]{2}{*}{ Não } & \multirow[t]{2}{*}{ RPaj $^{*}$} & \multicolumn{2}{|c|}{ IC95\% } \\
\hline Tabagismo & & & & & & & & & & & & & & & \\
\hline Fumante & 11,4 & 15,7 & 0,8 & 0,6 & 1,0 & 6,8 & 10,9 & 0,6 & 0,5 & 0,8 & 6,6 & 9,2 & 0,7 & 0,5 & 0,9 \\
\hline Ex-fumante & 27,9 & 29,7 & 0,8 & 0,5 & 1,4 & 16,4 & 17,0 & 0,6 & 0,4 & 0,9 & 16,5 & 13,5 & 0,6 & 0,4 & 1,0 \\
\hline $\begin{array}{l}\text { Consumo de } 20 \text { ou } \\
\text { mais cigarros }\end{array}$ & 4,1 & 5,6 & 0,9 & 0,8 & 1,0 & 1,8 & 3,0 & 0,9 & 0,8 & 1,0 & 1,3 & 1,9 & 1,0 & 0,8 & 1,2 \\
\hline $\begin{array}{l}\text { Fumantes passivos } \\
\text { no domicílio }\end{array}$ & 6,6 & 9,4 & 0,7 & 0,6 & 1,0 & 8,8 & 10,9 & 0,8 & 0,7 & 1,0 & 7,9 & 10,9 & 0,8 & 0,6 & 1,1 \\
\hline $\begin{array}{l}\text { Fumantes passivos } \\
\text { no trabalho }\end{array}$ & 9,1 & 10,2 & 1,0 & 0,7 & 1,3 & 7,0 & 9,3 & 0,8 & 0,6 & 0,9 & 5,2 & 5,9 & 1,0 & 0,7 & 1,3 \\
\hline $\begin{array}{l}\text { Reg. Frutas e } \\
\text { Hortaliças }\end{array}$ & 40,3 & 29,8 & 1,3 & 1,1 & 1,4 & 39,6 & 28,7 & 1,3 & 1,2 & 1,4 & 51,3 & 41,5 & 1,1 & 1,1 & 1,3 \\
\hline $\begin{array}{l}\text { Recom. de frutas e } \\
\text { hortaliças }\end{array}$ & 25,2 & 17,9 & 1,3 & 1,1 & 1,6 & 27,6 & 19,3 & 1,4 & 1,3 & 1,5 & 36,7 & 28,0 & 1,2 & 1,1 & 1,4 \\
\hline $\begin{array}{l}\text { Carnes com } \\
\text { excesso de gordura }\end{array}$ & 27,2 & 33,3 & 0,9 & 0,8 & 1,0 & 31,2 & 35,1 & 0,9 & 0,8 & 1,0 & 26,2 & 32,6 & 0,86 & 0,8 & 0,9 \\
\hline Leite com gordura & 46,4 & 53,7 & 0,9 & 0,8 & 0,9 & 53,0 & 60,0 & 0,9 & 0,8 & 0,9 & 41,3 & 52,9 & 0,8 & 0,8 & 0,9 \\
\hline $\begin{array}{l}\text { Refrigerantes ( } 5 \mathrm{x} \\
\text { ou mais/Sem) }\end{array}$ & 15,6 & 17,1 & 1,0 & 0,8 & 1,2 & 21,0 & 23,9 & 0,9 & 0,8 & 1,0 & 16,0 & 19,8 & 0,9 & 0,7 & 1,0 \\
\hline $\begin{array}{l}\text { Doces ( } 5 \mathrm{x} \text { ou mais/ } \\
\text { Sem) }\end{array}$ & 15,3 & 12,8 & 1,3 & 1,0 & 1,6 & 22,4 & 20,8 & 1,1 & 1,0 & 1,3 & 27,0 & 23,6 & 1,2 & 1,0 & 1,3 \\
\hline $\begin{array}{l}\text { feijão ( } 5 x \text { ou mais/ } \\
\text { Sem) }\end{array}$ & 65,4 & 70,7 & 0,9 & 0,9 & 1,0 & 66,3 & 68,4 & 1,0 & 0,9 & 1,0 & 53,4 & 62,8 & 0,9 & 0,8 & 0,9 \\
\hline $\begin{array}{l}\text { AF suficiente no } \\
\text { lazer }\end{array}$ & 31,7 & 22,8 & 1,5 & 1,3 & 1,7 & 43,5 & 37,1 & 1,2 & 1,1 & 1,3 & 50,4 & 47,3 & 1,1 & 1,0 & 1,2 \\
\hline Inatividade Física & 22,7 & 19,4 & 1,0 & 0,9 & 1,2 & 14,4 & 13,5 & 1,0 & 0,9 & 1,2 & 14,0 & 10,9 & 1,2 & 1,0 & 1,5 \\
\hline $\begin{array}{l}\mathrm{AF}>=150 \mathrm{~min} / \mathrm{sem} \\
\text { em } 3 \text { domínios }\end{array}$ & 43,9 & 44,2 & 1,1 & 1,0 & 1,2 & 57,4 & 54,0 & 1,1 & 1,1 & 1,2 & 59,2 & 58,4 & 1,1 & 1,0 & 1,1 \\
\hline $\begin{array}{l}\mathrm{AF}<150 \mathrm{~min} / \mathrm{sem} \\
\text { em } 3 \text { domínios }\end{array}$ & 56,1 & 55,8 & 0,9 & 0,9 & 1,0 & 42,6 & 46,0 & 0,9 & 0,9 & 1,0 & 40,8 & 41,6 & 0,9 & 0,9 & 1,0 \\
\hline $\begin{array}{l}\text { Hábito de assistir } \\
\text { TV - 3hrs/dia }\end{array}$ & 23,1 & 23,6 & 1,0 & 0,8 & 1,1 & 22,9 & 26,5 & 0,9 & 0,8 & 0,9 & 18,1 & 19,1 & 0,9 & 0,8 & 1,1 \\
\hline $\begin{array}{l}\text { Consumo abusivo } \\
\text { de álcool }\end{array}$ & 9,8 & 14,7 & 0,8 & 0,6 & 1,0 & 16,8 & 19,4 & 0,9 & 0,8 & 1,0 & 21,0 & 20,7 & 1,1 & 0,9 & 1,3 \\
\hline $\begin{array}{l}\text { Direção após } \\
\text { consumo de álcool }\end{array}$ & 2,9 & 3,1 & 1,1 & 0,6 & 2,0 & 5,2 & 5,3 & 0,9 & 0,7 & 1,2 & 9,6 & 6,9 & 1,4 & 1,1 & 1,8 \\
\hline Excesso de peso & 59,6 & 62,7 & 1,0 & 0,9 & 1,0 & 52,6 & 51,3 & 1,0 & 0,9 & 1,0 & 46,6 & 47,4 & 0,9 & 0,9 & 1,0 \\
\hline Obesidade & 18,8 & 25,7 & 0,7 & 0,6 & 0,9 & 17,5 & 18,1 & 0,9 & 0,8 & 1,1 & 14,5 & 15,0 & 0,9 & 0,7 & 1,1 \\
\hline $\begin{array}{l}\text { Avaliação ruim de } \\
\text { saúde }\end{array}$ & 6,8 & 8,0 & 0,8 & 0,6 & 1,2 & 3,0 & 4,4 & 0,7 & 0,5 & 0,9 & 2,3 & 3,5 & 0,7 & 0,5 & 1,1 \\
\hline $\begin{array}{l}\text { Mamografia há } 2 \\
\text { anos }\end{array}$ & 80,5 & 68,3 & 1,2 & 1,1 & 1,3 & 88,5 & 75,0 & 1,2 & 1,1 & 1,3 & 92,0 & 74,2 & 1,2 & 1,1 & 1,4 \\
\hline Papanicolau 3 anos & 83,9 & 75,6 & 1,1 & 1,0 & 1,2 & 86,4 & 75,9 & 1,1 & 1,1 & 1,2 & 86,6 & 81,2 & 1,0 & 1,0 & 1,1 \\
\hline Hipertensão & 43,1 & 38,6 & 1,0 & 0,9 & 1,1 & 18,5 & 17,9 & 0,87 & 0,8 & 0,9 & 15,4 & 14,0 & 0,8 & 0,7 & 1,0 \\
\hline Diabetes & 15,2 & 12,8 & 1,0 & 0,8 & 1,2 & 4,9 & 4,1 & 0,9 & 0,7 & 1,1 & 3,9 & 3,2 & 0,9 & 0,6 & 1,2 \\
\hline
\end{tabular}


temente associada com escolaridade, renda e melhores indicadores de saúde ${ }^{5,6}$.

O aumento da cobertura de planos de saúde após 55 anos de idade está coerente com outros estudos ${ }^{7,10,11}$ e também com os dados do Sistema de Informação de Beneficiários (SIB) da ANS ${ }^{23}$, o que tem sido justificado pela maior necessidade de consumo de serviços de saúde e maior demanda de cobertura entre idosos ${ }^{21,22}$.

As prevalências dos indicadores de tabagismo foram mais baixas na população com planos de saúde. Em geral, populações com mais escolaridade, renda e melhores condições socioeconômicas, têm maior acesso a informação sobre os malefícios do fumo e menores prevalências ${ }^{15,23}$. Destacam-se, que no estudo atual, as diferenças persistiram, mesmo ajustando por escolaridade.

A OMS recomenda o consumo diário de $400 \mathrm{~g} /$ dia de frutas e hortaliças para prevenção de doenças cardiovasculares, bem como redução do consumo de gorduras, açúcares e sal, que foram mais elevados na população com planos de saúde ${ }^{1,24}$. Os indicadores de consumo de frutas e hortaliças, consumo de gorduras e refrigerantes foram menores na população com planos, o que pode ser explicado pelo maior poder econômico para a aquisição de alimentos saudáveis como frutas, legumes e hortaliças, além de maior conhecimento sobre alimentos não saudáveis ${ }^{8,25}$. Exceção na população com planos foi o maior consumo de alimentos doces, e com planos e elevada escolaridade, foi o indicador de consumo de feijão, com menor prevalência, o que também foi identificado na $\mathrm{PNS}^{24,25}$. Possivelmente populações com mais renda têm diversificado sua alimentação e com isto reduzido o consumo de feijão, o que constitui uma perda, devido aos efeitos benéficos do mesmo, pelo aporte de fibras e nutrientes, resultando em maior saciedade e prevenção de obesidade ${ }^{26}$.

Também, a população com planos praticou mais atividade física no lazer, e nos demais domínios, sendo menos sedentária e com menor tempo assistindo TV. Estes indicadores positivos da atividade física têm sido explicados em populações de mais escolaridade e renda, pelo maior acesso a espaços para prática de atividade física, maior conhecimento sobre os benefícios da AF. Assim, torna-se importante investir em políticas públicas de melhorias de espaços públicos para prática de atividade física, visando reduzir estas desigualdades ${ }^{26}$.

A autoavaliação do estado de saúde ruim tem sido atribuída na literatura como importante indicador de saúde, preditor de mortalidade, em especial entre idosos, sendo usada internacionalmente $^{27}$. Em geral, pessoas com mais escolaridade, renda, posse de planos de saúde, jovens, com mais acesso a bens e serviços tendem a avaliar melhor seu estado de saúde, o que está em sintonia com os resultados aqui encontrados na população com planos de saúde ${ }^{27}$. Ao estratificar por escolaridade, em geral, permaneceu a melhor avaliação de saúde entre populações com planos, em especial na escolaridade média, 9 a 11 anos de estudo.

São preconizados na prevenção de câncer de colo uterino e de mama realizar o exame de Papanicolau a cada três anos entre mulheres de 25 a $64 \operatorname{anos}^{28}$ e o exame da mamografia a cada dois anos na faixa etária de 50 a $69 \operatorname{anos}^{28,29}$. As prevalências destes dois exames foram muito elevadas em ambas as populações, mas ainda mais na população com planos de saúde. Destaca-se que a meta prevista no Plano de Enfrentamento de Doenças Crônicas para 2022 é atingir 75\% em mamografia ${ }^{30}$, já superada na população com planos de saúde, mas ainda abaixo entre mulheres sem planos de saúde. Também para a citologia oncótica (Papanicolau) a meta do Plano de Enfrentamento de DCNT de alcançar cobertura média de $85 \%$ em 2022 já foi superada na população com planos de saúde e praticamente atingida na população coberta apenas pelo SUS $(84,7 \%)^{30}$, apontando a importância da Atenção Primária no Brasil, que oferta amplamente este exame pelo Sistema Único de Saúde (SUS) 9 . Destacamos ainda que as diferenças na população com e sem planos de saúde persistem quando estratificadas por escolaridade.

Nos Estados Unidos, mulheres que têm planos de saúde tiveram maiores coberturas de mamografia e citologia oncótica, o que poderia ser explicado pelo maior acesso aos serviços diagnósticos e preventivos nessa população $0^{13,14}$.

$\mathrm{O}$ excesso de peso e a obesidade estão associados às doenças cardiovasculares, diabetes, câncer de cólon, reto e de mama, cirrose, dentre outras ${ }^{1,2}$, constituindo um grande problema global a ser enfrentado, dado o seu crescimento continuo na maioria dos países ${ }^{31}$, constituindo meta do plano Nacional ${ }^{30}$ e do Plano Global de NCD deter o seu crescimento ${ }^{32}$. Embora seja um problema disseminado, a população com planos de saúde mostrou menor chance de ter excesso de peso e obesidade.

Estudo sobre hipertensão arterial (HA) e fatores associados, analisando dados do Vigitel, apontou que, após a análise multivariada e ajuste por todas as variáveis do modelo, ter planos de saúde deixou de ser fator de proteção de 
$\mathrm{HA}$, indicando que a escolaridade constitui-se na variável socioeconômica de maior relevância na "redução da hipertensão"33. O estudo atual mostrou ter planos como fator de proteção para HA quando ajustado por sexo, idade e escolaridade, entretanto, na análise estratificada por três níveis de escolaridade, ter planos só se mostrou protetor entre 9 e 11 anos de escolaridade. Além disto, a prevalência de HA na população com planos e baixa escolaridade foi três vezes mais elevada, quando comparada com a população com planos e alta escolaridade, confirmando a importância da escolaridade como fator de proteção para a HA.

Ter diabetes não se mostrou associado com planos de saúde, entretanto, populações com baixa escolaridade têm até quatro vezes mais diabetes, comparada com as de 12 anos e mais de escolaridade. Também em estudos internacionais, como o Alameda County Study, publicado em 2005 por Maty et al. ${ }^{34}$, apontaram associação entre o nível educacional e o diabetes mellitus, após o ajustamento para as variáveis renda e ocupação. Isto sugere que a escolaridade, considerada proxy de nível socioeconômico, se associe a mais acesso a práticas de promoção a saúde, como alimentação saudável, atividade física, acesso a medicamentos e serviços de saúde ${ }^{35}$.

O consumo abusivo de álcool não teve diferenças segundo a posse de planos, mas foi mais prevalente em populações de elevada escolaridade, o que está coerente com outros estudos do Vigitel $^{17}$. Quando estratificado por escolaridade, o consumo de álcool e a direção foi mais frequente em ter planos de saúde e escolaridade elevada, o que pode se justificar pela posse de carro estar relacionada ao melhor status socioeconômico.

O estudo aponta iniquidades em saúde, melhores indicadores na população com acesso a planos de saúde, reflexo da escolaridade mais elevada, acesso mais facilitado a serviços de saúde e práticas de promoção. Desta forma, investir na melhoria de práticas educativas, políticas públicas de promoção à saúde é essencial para reduzir estas desigualdades.

Dentre os limites do estudo, destacam-se o uso de entrevistas telefônicas, o que pode reduzir a participação de populações sem telefonia fixa. Busca-se reduzir esse viés utilizando pesos de pós -estratificação. O fato de que as informações são autoreferidas também pode resultar em viés de informação, embora a experiência nacional e internacional aponte que variáveis como hipertensão arterial e avaliação de estado de saúde conseguem obter boas estimativas usando esta metodologia, além de apresentar vantagens como rapidez na informação, sensibilidade e baixo custo ${ }^{18}$. Além disso, o delineamento transversal do estudo não permite estabelecer relação temporal de causa e efeito. Assim, não se pode afirmar que o acesso aos planos de saúde resulte em menor exposição a risco ou se indivíduos mais preocupados com a própria saúde procurariam os planos de saúde.

\section{Conclusão}

O estudo mostrou diferenças nos fatores de risco e proteção entre adultos filiados ou não a plano privado de saúde, sendo possível observar hábitos mais saudáveis entre os primeiros, como consumo de frutas e hortaliças, prática de atividade física e menor prevalência de tabagismo e álcool abusivo. A prevalência de uso de exames preventivos foi, também, significativamente mais alta entre os filiados a plano privado, em comparação àqueles que dependem exclusivamente do SUS, mesmo estratificando-se esses resultados pela escolaridade. A morbidade referida não diferiu segundo ter planos de saúde. Monitorar os fatores de risco de DCNT é importante para apoiar políticas públicas de prevenção. 


\section{Colaboradores}

DC Malta participou da concepção do estudo, do delineamento do estudo; aquisição, análise e interpretação dos dados do trabalho e elaboração da primeira versão do artigo. RTI Bernal participou da concepção do estudo, do delineamento do estudo; aquisição, análise estatística e interpretação dos dados do trabalho; e revisão crítica; E Vieira Neto, KA Curci, RM Lisbôa, RF Cachapuz, MIF Freitas, KSC Coelho, FP Santos, MT Marcilacc participaram da análise e interpretação dos dados do trabalho, revisão crítica, e todos os autores aprovaram a versão final a ser publicada; concordaram em ser responsáveis por todos os aspectos do trabalho, no sentido de garantir que as questões relacionadas à exatidão ou à integridade de qualquer parte da obra sejam devidamente investigadas e resolvidas.

\section{Agradecimentos}

Os autores agradecem ao Ministério da Saúde, Secretaria de Vigilância em Saúde, Agência Nacional de Saúde Suplementar. Malta DC agradece ao CNPq pela bolsa de produtividade em pesquisa.

\section{Referências}

1. World Health Organization (WHO). Global status report on noncommunicable diseases 2014. Geneva: WHO; 2014. [acessado 2017 jul 18]. Disponível em: http://apps.who.int/iris/bitstream/106 65/148114/1/9789241564854_eng.pdf?ua=1

2. World Economic Forum and World Health Organization (WHO). From burden to "best buys": reducing the economic impact of non-communicable diseases in low- and middle-income countries. Geneva: World Economic Forum; 2011. [acessado 2017 Jul 18]. Disponível em: http://www.who.int/nmh/publications/ best_buys_summary.pdf?ua $=1$

3. Abegunde DO, Mathers CD, Adam T, Ortegon M, Strong K. The burden and costs of chronic diseases in low-income and middle-income countries. Lancet 2007; (370):1929-1938.

4. Barros MBA, Lima MG, Medina LPB, Szwarcwald CL, Malta DC. Social inequalities in health behaviors among Brazilian adults: National Health Survey, 2013. Int J Equity Health 2016; 15:148.

5. Malta DC, Bernal RTI, Souza MFM, Szwarcwald CL, Lima MG, Barros MB. Social inequalities in the prevalence of self-reported chronic non-communicable diseases in Brazil: national health survey 2013. Int J Equity Health 2016; 15(1):153.

6. Malta DC, Bernal RTI, Lima MG, Araújo SSC, Silva MMA, Freitas MIF, Barros MBA. Noncommunicable diseases and the use of health services: analysis of the National Health Survey in Brazil. Rev Saude Publica 2017; 51(Supl. 1):4s.

7. Instituto Brasileiro de Geografia e Estatística (IBGE). Pesquisa Nacional por Amostra de Domicílios (PNAD 2008). Um panorama da saúde no Brasil: acesso e utilização dos serviços, condições de saúde: 2008. Rio de Janeiro: IBGE; 2010. [acessado 2017 Jul 18]. Disponível em: https://biblioteca.ibge.gov.br/visualizacao/ monografias/GEBIS\%20-\%20RJ/panorama.pdf

8. Valle EA, Mambrini JVM, Macinko J, Lima-Costa MF. Comportamentos em saúde e exames preventivos entre adultos filiados ou não a planos de saúde na Região Metropolitana de Belo Horizonte, Minas Gerais, Brasil, 2003-2010. Cad Saude Publica 2017; 33(3):e00130815.

9. Stopa SR, Malta DC, Monteiro CN, Szwarcwald CL, Goldbaum Moisés, Cesar CLG. Acesso e uso de serviços de saúde pela população brasileira, Pesquisa Nacional de Saúde 2013. Rev Saude Publica 2017; 51(Supl. 1):3s.

10. Instituto Brasileiro de Geografia e Estatística (IBGE). Pesquisa Nacional de Saúde 2013: percepção do estado de saúde, estilos de vida e doenças crônicas. Rio de Janeiro: IBGE; 2014. [acessado 2017 Jul 18]. Disponível em: ftp://ftp.ibge.gov.br/PNS/2013/pns2013.pdf

11. Malta DC, Stopa SR, Pereira CA, Szwarcwald CL, Oliveira M, Reis AC. Cobertura de Planos de Saúde na população brasileira, segundo a Pesquisa Nacional de Saúde, 2013. Cien Saude Colet 2017; 22(1):179-190.

12. Silva GA, Souza-Júnior PRB, Damacena GN, Szwarcwald CL. Detecção precoce do câncer de mama no Brasil: dados da Pesquisa Nacional de Saúde, 2013. Rev Saude Publica 2017; 51(Supl. 1):14s. 
13. Ahluwalia JB, Bolen J, Garvin B. Health insurance coverage and use of selected preventive services by working age women, BRFSS 2006. J Womens Health (Larchmt) 2007; 16(7):935-940.

14. Nelson KM, Chapko MK, Reiber G, Boylo EJ. The association between health insurance coverage and diabetes care; data from the 2000 behavior risk factor surveillance system. Health Serv Res 2005; 40(2):361372.

15. Malta DC, Bernal RTI. Comparação dos fatores de risco e proteção de doenças crônicas na população com e sem planos de saúde nas capitais brasileiras, 2011. Rev. bras. epidemiol. 2014; 17(Supl. 1):241-255.

16. Brasil. Ministério da Saúde (MS). VIGITEL Brasil 2016: vigilância de fatores de risco e proteção para doenças crônicas por inquérito telefônico. Brasília: MS; 2017. [acessado 2017 Jul 18]. Disponível em: http://portalarquivos.saude.gov.br/images/pdf/2017/junho/07/ vigitel_2016_jun17.pdf

17. Brasil. Ministério da Saúde (MS), Vigitel Brasil 2015 Saúde Suplementar: vigilância de fatores de risco e proteção para doenças crônicas por inquérito telefônico. Brasília: MS; 2017. [acessado 2017 Jul 18]. Disponível em: http://portalarquivos.saude.gov.br/ images/pdf/2017/marco/07/vigitel_saude_suplementar_2015.pdf

18. Kalton G, Flores-Cervantes I. Weighting methods. J Off Stat 2003 [acessado 2017 Set 15];19(2):81-97. Disponível em: http://www.jos.nu/articles/abstract. asp?article $=192081$

19. Izrael D, Hoaglin, DC, Battaglia MP. A SAS Macro for Balancing a Weighted Sample. In: Proceedings of the Twenty-Fifth Annual SAS Users Group International Conference, 2000 Apr 9-12, Paper 275, Cary (NC):SAS Institute; 2000 [acessado 2017 Jul 18]. Disponível em: http://www2.sas.com/proceedings/sugi25/25/ st/25p258.pdf

20. Barros AJ, Hirakata VN. Alternatives for logistic regression in cross-sectional studies: an empirical comparison of models that directly estimate the prevalence ratio. BMC Medical Research Methodology 2003; $3: 21$.

21. Agência Nacional de Saúde Suplementar. Caderno de Informações de Saúde Suplementar: Beneficiários, Operadoras e Planos. Rio de Janeiro: ANS; ano 11, n. 1 (jun.) 2017. [acessado 2017 Set 15]. Disponível em: http://www.ans.gov.br/images/stories/Materiais_para _pesquisa/Perfil_setor/Caderno_informacao_saude _suplementar/caderno_informacao_junho_2017.pdf

22. Lima-Costa MF, Guerra HL, Firmo JO, Vidigal PG, Uchoa E, Barreto SM. The Bambuí Health and Ageing Study (BHAS): private health plan and medical care utilization by older adults. Cad Saude Publica 2002; 18(1):177-186

23. Giovino GA, Mirza SA, Samet JM, Gupta PC, Jarvis MJ, Bhala N, Peto R, Zatonski W, Hsia J, Morton J, Palipudi KM, Asma S, GATS Collaborative Group. Tobacco use in 3 billion individuals from 16 countries: an analysis of nationally representative cross-sectional household surveys. Lancet 2012; 380:(9842):668-679.
24. Jaime PC, Stopa SR, Oliveira TP, Vieira ML, Szwarcwald CL, Malta DC. Prevalência e distribuição sociodemográfica de marcadores de alimentação saudável, Pesquisa Nacional de Saúde, Brasil 2013. Epidemiol. Serv. Saúde 2015; 24(2):267-276.

25. Velásquez-Meléndez G, Mendes LL, Pessoa MC, Sardinha LMV, Yokota RTC, Bernal RTI, Malta DC. Tendências da frequência do consumo de feijão por meio de inquérito telefônico nas capitais brasileiras, 2006 a 2009. Cien Saude Colet 2012; 17(12):3363-3370.

26. Simões EJ, Hallal PC, Siqueira FV, Schmaltz C, Menor D, Malta DC, Duarte H, Hino AA, Mielke GI, Pratt M, Reis RS. Effectiveness of a scaled up physical activity intervention in Brazil: A natural experiment. Prev Med 2017; 103S:S66-S72.

27. Molarius A, Berglund K, Eriksson C, Lambe M, Nordström E, Eriksson H, Feldman I. Socioeconomic conditions, lifestyle factors, and self-rated health among men and women in Sweden. Eur J Public Health 2007; 17(2):125-133.

28. Instituto Nacional do Câncer (INCA). Programa Nacional de Controle do Câncer do Colo do Útero [Internet]. [acessado 2017 Set 15]. Disponível em: http:// www2.inca.gov.br/wps/wcm/connect/acoes_programas/site/home/nobrasil/programa_nacional_controle_cancer_colo_utero

29. Instituto Nacional do Câncer (INCA). Programa Nacional de Controle do Câncer de Mama [Internet] [acessado 2017 Set 15]. Disponível em: http://www2. inca.gov.br/wps/wcm/connect/acoes_programas/site/ home/nobrasil/programa_controle_cancer_mama

30. Malta DC, Morais Neto OL, Silva Junior JB. Apresentação do plano de ações estratégicas para o enfrentamento das doenças crônicas não transmissíveis no Brasil, 2011 a 2022. Epidemiol. Serv. Saúde 2011; 20(4):425-438.

31. International Association for the Study of Obesity (IASO) (2013) Adult overweight and obesity in the European Union (EU27). [acessado 2013 nov 02]. Disponível em: http://www.iaso.org/resources/world -map-obesity/ Accessed novembro 2017.

32. World Health Organization (WHO). Global action plan for the prevention and control of NCDs 2013-2020. Geneva: WHO; 2013. [acessado $2017 \mathrm{Jul}$ 18]. Disponível em: http://apps.who.int/iris/bitstream/10665/94384/1/9789241506236_eng.pdf?ua=1

33. Malta DC, Bernal RTI, Andrade SSCA, Silva MMA, Velasquez-Melendez G. Prevalência e fatores associados com hipertensão arterial autorreferida em adultos brasileiros. Rev Saude Publica 2017; 51(Supl. 1):11s.

34. Maty SC, Everson-Rose SA, Haan MN, Raghunathan TE, Kaplan GA. Education, income, occupation, and the 34-year incidence (1965-99) of Type 2 diabetes in the Alameda County Study. Int J Epidemiol 2005; 34(6):1274-1281.

35. Malta DC, Bernal RTI, Iser BPM, Szwarcwald CL, Duncan BB, Schmidt MI. Fatores associados ao diabetes autorreferido segundo a Pesquisa Nacional de Saúde, 2013. Rev Saude Publica 2017; 51(Supl. 1):12s.

Artigo apresentado em 16/09/2018

Aprovado em 03/12/2018

Versão final apresentada em 05/12/2018 
